\title{
Knowledge of Oral Cancer and Screening Practices of Primary Care Providers at Federally Qualified Health Centers
}

\author{
Woosung Sohn, DDS, PhD, DrPH ; Amid I. Ismail, BDS, MPH, DrPH; Justine L. Kolker, DDS, MS, \\ PhD
}

\begin{abstract}
Objectives: Primary care providers (PCPS) who worked in Federally-Qualified Health Centers (FQHC) in Michigan were surveyed to assess their knowledge level and practices related to screening and preventing oral cancer. Methods: $A$ questionnaire was developed with the assistance of dental and medical experts, and revised through focus groups. The questionnaire included one case scenario describing a suspicious oral lesion in a 55-year old female patient, followed by questions assessing PCPs' knowledge level, attitude, opinion, and screening practices for oral cancer. This mail survey was conducted in 2003. Results: Survey response rate was $56.4 \%$. Over $70 \%$ of the respondents reported that they screen patients for oral cancer during a routine physical examination. Forty-four percent of $P C P$ s had high knowledge level, based on the scenario questions. Those who had high knowledge level were more likely to be physicians, males, and more likely to perform screening for oral cancer than those with low knowledge level. There was no difference in age and race/ethnicity between high and low knowledge groups. Perceived barriers included (1) lack of education; (2) lack of specialists to refer patients; and (3) lack of reimbursement. Conclusions: The majority of PCPs in this survey had positive attitudes about performing screening for oral cancer. To involve $P C P$ s in screening for oral cancer, oral health programs should focus on providing up-to-date education, setting up a referral system, and providing proper reimbursement.
\end{abstract}

Key words: oral cancer screening, prevention, early diagnosis, primary care provider, survey

\section{Introduction}

Healthy People 2010 (1) advocated for an increase to $50 \%$ of oral and pharyngeal cancer to be detected at the earliest stage (rates in 1998: 21\% for African Americans (AA) and 38\% for white Americans (WA)); an increase to $20 \%$ of adults who in the past 12 months reported having had an examination to detect oral and pharyngeal cancers (rates in 1998 were $7 \%$ for AA and $14 \%$ for WA); and a decrease of the oropharyngeal cancer death rate to 2.7 deaths per 100,000 in 2010 (rates in 1998 were 4.5 for AA and 2.4 for WA). One way to achieve these goals is to develop a network of dental and other healthcare providers to promote the early screening of oral soft tissue lesions that may be precancerous or cancerous $(2,3)$. Unfortunately, for a large segment of high-risk individuals, access to dental care is limited, however, there is relatively better access to nondental primary health care providers (PCPs) $(4,5)$. Therefore, PCPs could play a major role in prevention of oral cancer and reducing death due to oral cancer $(6,7)$. PCPs could also screen tobacco users for early signs of oral cancer and counsel patients to reduce the exposure to tobacco products and excessive drinking of alcohol $(8,9)$. As a preventive guideline, the American Cancer Society recommends an annual oral cancer screening for adults 40 years of age and older (10).
Also, the US Preventive Services Task Force recommends an annual oral cancer screening for tobacco and alcohol users (11). Therefore, oral cancer screening is expected to be included in PCPs' services to their patients who are 40 years and older or tobacco and alcohol users.

An important question that needs to be answered regarding screening for oral cancer by PCPs is whether they can or are willing to perform screening for oral cancer. This expanded role may require development of programs to train as well as to compensate PCPs for taking an active role in screening for oral cancer. While there are many studies that report the knowledge level, oral cancer screening practices, and attitude and barriers to perform oral cancer screenings of physicians, dentists, dental hygienists (12-16), there is limited knowledge about the oral cancer screening practices of primary care providers especially those who provide health care to underserved populations.

This paper presents findings of a survey of PCPs who worked for Federally-Qualified Health Centers (FQHCs) and community-based clinics in Michigan. These PCPs provide health care services to over 270,000 underserved residents of Michigan. The purpose of this study was to assess their attitude and knowledge level on oral cancer diagnosis and prevention; screening practices for oral cancer; and barriers and incentives that may hinder or promote screening for oral cancer.

Send correspondence and reprint requests to Woosung Sohn, DDS, PhD, DrPH, Department. of Cariology, Restorative Sciences \& Endodontics, School of Dentistry, University of Michigan, 1101 N. University, Ann Arbor, MI 48109-1078. E-mail: woosung@umich.edu. Dr. Sohn is an Assistant Professor in the Department of Cariology, Restorative Sciences and Endodontics, School of Dentistry, University of Michigan. Dr. Ismail is a Professor in the Department of Cariology, Restorative Sciences and Endodontics, School of Dentistry, University of Michigan. Dr. Kolker is an Assistant Research Scientist/Clinical Assistant Professor in the Department of Cariology, Restorative Sciences and Endodontics, School of Dentistry, University of Michigan. Manuscript received 8/11/04; returned to authors for revision 3/01/04; final version accepted for publication $4 / 6 / 05$. This project is funded by the National Institute of Dental and Craniofacial Research / National Institutes of Health Grant DE 14410-03. 
Methods

Questionnaire development. The Michigan Oral Cancer Prevention Network (MOCPN) research team developed a questionnaire to evaluate attitudes and knowledge about oral cancer as well as screening practices of PCPs. The questionnaire also included questions regarding barriers and incentives that might hinder or promote screening for oral cancer. The content of the questionnaire was developed with the assistance of dental and medical experts. The questionnaire was then refined through a series of three focus group and revision sessions. Fourteen PCPs participated in the focus groups; each PCP attended only one focus group session. The questionnaire included one case scenario (with photograph) describing a suspicious oral lesion (Figure $1)$.

After the description of the case scenario, the respondents were presented with questions asking about their knowledge of diagnostic signs of oral cancer, signs of metastasis, and screening procedure for oral cancer.

Mail survey. The MOCPN, in collaboration with the Michigan Primary Care Association (MPCA) which represents and coordinates the activities of all FQHCs in the state, developed a database that listed all $28 \mathrm{FQHC}^{\prime}$ s and all the PCPs affiliated with the MPCA. The survey was sent to all 303 PCPs who were currently employed by the FQHCs.

Each $\mathrm{PCP}$ received a personalized cover letter, a colored map depicting county level incidence rates of oral cancer in Michigan, a questionnaire, and a self-addressed and stamped envelope. The first mailing was sent on January 21,2003 followed by a reminder card that was mailed on February 14,2003 . A second mailing was sent to the sampled providers who did not respond by March 20, 2003. Nonrespondents received a third mailing on May 2, and a final mailing was sent on July 7, 2003.

Statistical analysis. Returned questionnaires were coded and entered using EpiData software (The EpiData Association, Odense Denmark, ver 3, released 2003) (17). After data entry, statistical analysis was conducted using the SAS programs for descriptive and multivariate analyses.

Correct answers for the eight questions related to the case scenario were obtained from an expert in Oral Medicine in the University of Michigan, School of Dentistry (Dr. Sharon Brooks). The knowledge level of PCPs was determined by number of correct responses to the 8 case scenario ques-

\section{FIGURE 1}

Case scenario of suspicious oral lesion

We would like your opinion on the management of the following patient:

Age: 55

Sex: Female

Smoking history: Alcohol use:

\section{Never smoked.}

Initially she said that she is a religious person and does not drink; however, after further questioning she indicated that she drinks in the evening each day before going to bed. She said that she does not want anyone to know that she drinks.

Family history: Sister was diagnosed with lymphoma at the age of 22 . She died 2 years later.

Health history: Patient reported that she was diagnosed with depression and was treated for a period of 2 years.

Marital status: $\quad$ Divorced; children left home after high school.

Chief complaint: Swelling on the side of the tongue that was felt by the patient 3 months ago. However, it was diagnosed by another physician as a traumatic swelling due to tongue biting that presumably occurred during a dinner with a friend.

Clinical condition: Refer to the picture at right

tions. PCPs were classified with "high knowledge score (HKS)" when they answered at least six of the eight items correctly (i.e., at least 75 percent of the items were correct). Providers who answered less than six items correctly were labeled with "low knowledge score (LKS)".

Bivariate and multivariate analyses were conducted to identify the PCPs' characteristics (e.g., practice behaviors, attitudes, and background characteristics such as demographics) in the HKS group in comparison with those in the LKS group.

\section{Results}

Of the 303 PCPs listed in the database, a total of 171 PCPs responded to the survey (response rate $=56.4 \%$ ). Of the 171 respondents, nine were administrators and did not answer the questionnaire. Therefore this analysis included 162 completed questionnaires. Characteristics of the PCPs who responded were presented in Table 1.

Current oral cancer screening practices. Over $70 \%$ of the respondents reported that they screen patients for oral cancer during a routine physical examination (Table 2). Additionally, over two-thirds of the respondents $(66 \%)$ referred patients to a dentist or ENT specialist in the last 12 months for a suspicious oral cancer lesion. Although a high percent of respondents reported performing screening for oral cancer, only about a third $(36 \%)$ of the respondents felt that they were adequately trained to detect oral cancer lesions. Respondents who answered that they performed oral cancer screening also answered questions about the basis on which they select patients for screening. Many of the respondents reported that they select patients for oral cancer screen-

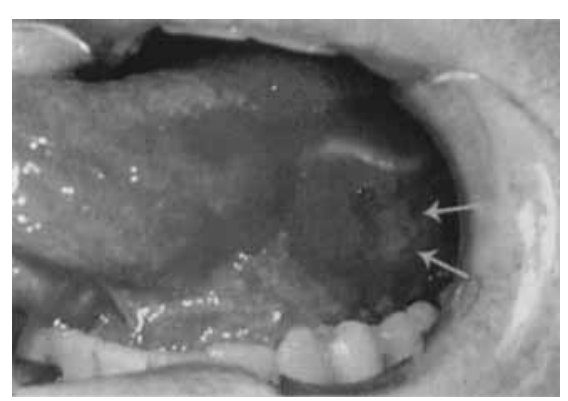


TABLE 1

Characteristics and background of respondents (\%)

\begin{tabular}{lr}
\hline $\begin{array}{l}\text { Responded PCPs in FQHCs } \\
\text { in Michigan (n=162) }\end{array}$ & $\%$ \\
\hline $\begin{array}{l}\text { Profession } \\
\text { Physician }\end{array}$ & 48.8 \\
Nurse practitioner/RN & 24.7 \\
Physician assistant & 21.6 \\
$\quad$ Other & 4.9 \\
Gender & \\
Female & 60.5 \\
Male & 39.5 \\
Race/Ethnicity & \\
White & 76.6 \\
Asian & 13.8 \\
African American & 5.5 \\
American Indian & 2.1 \\
Hispanic & 2.1 \\
Age & \\
29 and younger & 6.1 \\
$30-39$ & 34.5 \\
$40-49$ & 35.1 \\
$50-59$ & 23.0 \\
60 and older & 1.4 \\
\hline
\end{tabular}

ing based upon tobacco (94\%) and heavy alcohol use $(82 \%)$. Prior history of oral cancer $(90 \%)$ and family history of cancer (76\%) were also considered by many providers. Over half of the PCPs responded that age of the patient $(62 \%)$ and poorly fitting dentures $(52 \%)$ were factors they may have used to determine whether a patient should be screened for oral cancer.

Opinions/perceived barriers on oral cancer screening. Most respondents (92\%) agreed that annual oral cancer examinations should be performed for adults aged 40 years and older. When asked if tobacco users should have an annual examination regardless of age, nearly all of the respondents $(96 \%)$ either agreed or strongly agreed. Nearly half $(48 \%)$ of the respondents agreed or strongly agreed that oral cancer exams should be a separate reimbursable procedure (Table 2).

The most prominent barrier for oral cancer screening was the lack of adequate training ( $64 \%)$, followed by shortage of specialist to whom they could refer patient $(48 \%)$ and lack of time $(15 \%)$. Accordingly, majority of respondents answered that they needed more education about oral

TABLE 2

Current practice and opinions regarding oral cancer screening

\begin{tabular}{lc}
\hline & $\begin{array}{c}\text { PCPs who } \\
\text { answered "Yes" } \\
\mathbf{( \% )}\end{array}$ \\
Oral cancer screening practice & \\
Do you screen patients seen during a physical examination & 71.0 \\
$\quad$ for signs of oral cancer? & \\
In the past 12 months, have you referred patients to a dentist, an & \\
ENT specialist, or a surgeon for further diagnosis of suspicious & 66.4 \\
oral cancer lesions? & \\
Do you select patients for screening based upon: & 93.5 \\
Heavy use of tobacco products & 89.6 \\
Prior oral cancer lesion & 82.2 \\
Heavy alcohol use & 75.7 \\
Family history of cancer & 62.2 \\
Old age & 51.5 \\
Poorly fitting dentures & 40.7 \\
Sun exposure & 22.0 \\
Low consumption of fruits and vegetables &
\end{tabular}

Attitude/opinions/perceived barriers on oral cancer screening*

Oral cancer examinations for adults aged 40 years and older should be provided annually

91.7

Oral cancer examinations for tobacco users should be provided annually regardless of age

95.5

Oral cancer examination should be a separate reimbursable procedure 47.8

Primary care providers do not have time to screen for oral cancer $\quad 15.3$

Do you feel that you were adequately trained in medical school to detect oral cancer lesions?

In my geographic area, there is a shortage of specialists to whom I can refer patients with suspicious oral lesion

Would you be willing to participate in a network to promote early screening for oral cancer?

Programs or incentives to increase oral cancer screening ${ }^{+}$

More continuing education through $\mathrm{CME}$

More education in medical school

Setting up a referral system including dentist, ENT, cancer specialist, and PCP

51.0

Proper reimbursement

47.4

Development of better screening tools

42.9

* Percent of PCPs who answered "Agree or Strongly agree"

${ }^{+}$Respondents were allowed to choose all answers that applied

cancer screening either through continuing medical education $(76 \%)$ or education in medical school (65\%) for programs or incentives to promote oral cancer screening. Over half of the respondents (51\%) answered that setting up a referral system would also increase screening for oral cancer. Forty-five percent of the respondents were willing to participate in a network to promote early screening for oral cancer (Table 2).

Sources of information and internet and technology use. The most preferred information sources for
PCPs, in descending order, were $\mathrm{CME}$, presentations at professional meetings, articles in health journals, and educational mailing from professional organizations (Table 3). Nearly $85 \%$ of the PCPs answered that they were likely to learn from educational mailings sent directly by professional medical organizations or societies. Relative to the traditional methods, new communication methods such as email, websites, or CD-ROMs were preferred by smaller number of PCPs. Most respondents answered that they have had access to the Internet at 
TABLE 3

Preferred information sources and current technology use

$\begin{array}{lc}\text { Information source } & \% \\ \text { Continuing Medical Education } & 89.0 \\ \text { Presentations at professional meetings } & 86.8 \\ \text { Articles in health journals } & 84.7 \\ \text { Educational mailings sent directly by professional medical } & \\ \quad \text { organizations or societies } & 83.9 \\ \text { Email updates } & 48.6 \\ \text { CD-ROMs } & 47.9 \\ \text { A web site } & 46.2\end{array}$

Internet and technology use in patient care ${ }^{t}$

Do you have access to the internet in you office at work?

Do you have access to the internet at home?

93.0

Do you have technical person who trouble-shoot computer problems at your center or clinic?

80.4

Do you have access to digitized patents' records in your clinic?

14.1

Do you use computer to access any information on patients?

43.0

* Percent of PCPs who answered "Likely or Very likely" to use

+ Percent of PCPs who answered 'Yes'

home $(93 \%)$ and at work (87\%). Technical assistance was available to majority $(80 \%)$ of providers in their work. While almost half of respondents use a computer to retrieve some patient information, only $14 \%$ of them answered that they have access to digitized patient records in their clinic.

Knowledge level on oral cancer from a case scenario. For the questions regarding the presented casescenario, over $90 \%$ of the respondents agreed with a specialist in Oral Medicine that an ulceration that did not heal $(99 \%)$ and lymph gland involvement $(91 \%)$ are relevant in diagnosing a suspicious oral cancer lesion (Table 4). Three-fourths of the respondents agreed with the expert that the volume of alcohol consumption per day is relevant in diagnosing a suspicious oral lesion. Half of the respondents agreed with the expert in that the size of the swelling is relevant to determine the staging of a primary oral cancer. While $60 \%$ of the respondents reported that bleeding associated with the swelling was relevant in determining the diagnosis, the expert answered it was not; only $18 \%$ of the respondents agreed with the expert's answer. Eighty percent of respondents correctly answered that the presence of hard, painless, and fixed lymph nodes indicated the oral cancer had metastasized. While $89 \%$ of the respondents correctly answered how to examine the tongue for oral cancer screening, nearly half of the respondents $(45 \%)$ were not aware of which area of the tongue was most likely to develop oral cancer (Table 4).

Forty-four percent of PCPs were labeled as "high knowledge score $(\mathrm{HKS}) "$ and the remaining $56 \%$ of PCPs were labeled as "low knowledge score (LKS)" (Table 5). PCPs in the HKS group were more likely to be males, physicians, and regularly perform screening for oral cancer, compared with those in the LKS group. The HKS group was also more likely to answer that they were adequately trained to detect oral cancer lesions and that they were willing to participate in a network to promote early screening for oral cancer. There was no difference in age and race/ ethnicity between HKS and LKS groups (Table 5). PCPs in the HKS group were not different from those in the LKS group regarding barriers, incentives, preferred information sources, and internet and technology use.

\section{Discussion}

Prevention of cancer, including oral cancer, and reducing cancer-related burden and deaths could be achieved by an integrated program that involves health care providers, health care organizations, the government at various levels, and the public. We surveyed PCPs who work in Michigan FQHCs to assess their knowledge and screening practice for oral cancer. The survey response rate of $56.4 \%$ was achieved through four mailings and two reminder postcards. This response rate is slightly higher or at least on par with those reported in recent surveys of oral health issues among physicians (9, 18). We did not analyze non-responses for bias, however, there is always potential selection bias among respondents because they might be more interested in the topic of survey. The findings in this survey might not be generalized to primary care providers in non-FQHC and communitybased clinical settings, due to differences in the way care is provided in private and government supported clinics.

This survey found that primary care providers who work in FQHCs have positive attitudes about performing oral cancer screening in their clinics and would like to take an active part in a network to screen for oral cancer. These findings are encouraging since these PCPs provide care to under-served populations in rural or inner-city areas, where access to a dentist is often limited and individuals are more likely to have high-risk behaviors such as tobacco and alcohol use (8). In effort to reduce oral cancer mortality in under-served populations, it would be advantageous if FQHC PCPs screened for oral cancer in individuals with risk factors.

Our survey results found that many of these PCPs, in general, were knowledgeable about oral cancer and risk factors, and that PCPs who were more knowledgeable were more likely to perform oral cancer screening and/ or they are willing to perform screening in the future. These findings agree with a previous report which found that medical providers who are aware of risk factors refer more often than providers who are unaware of risk factors (19).

Although a high proportion (71\%) of the respondents answered that they 
TABLE 4

Case scenario: diagnostic points of suspicious oral cancer lesion

\section{Question statement}

Answer Choices*

PCPs' answers

Presence of ulceration that has not healed since the biting incident Relevant

Not relevant

98.7

0.6

Do not know

0.6

Whether there is bleeding associated with the swelling

Relevant

Not relevant

60.3

18.0

21.8

Volume of alcohol consumption per day

Relevant

75.6

Not relevant

Do not know

Lymph gland involvement to determine the standing of primary lesion

Relevant

90.5

Not relevant

Size of the swelling to determine the staging of primary lesion

Relevant

Not relevant

In this patient which of the following findings in the neck lymph nodes indicate that oral cancer has metastasized

Hard, painful, mobile

Hard, painless, fixed

79.8

Soft, painful, mobile

1.3

Soft, painless, fixed or mobile

2.5

Do not know

14.6

Which area of the tongue is MOST LIKELY to develop oral cancer?

All areas of the tongue

Dorsal surface

Ventral-lateral border

29.6

Anterior-lateral border

5.7

Base of tongue

10.1

Do not know

44.7

When examining the tongue of this patient, you should:

Have her stick out tongue as far as possible for inspection 0.6

Examine the posterior dorsum of the tongue with a tongue blade or mirror

Pull the patient's tongue out and inspect both sides of it

Inspect underside of the tongue by having the patient raise tongue

All of the above

Do not know

* Bolded choices indicate correct answer for each question, determined by an oral medicine expert.

screened for oral cancer, many respondents pointed out that the lack of sufficient training and proper referral system were major barriers to the screening practice. These findings are similar to a study among general medical practitioners; $91 \%$ wanted more training in detection of oral cancer and $67 \%$ wanted advice on referral pathways (20). Another barrier was lack of compensation for oral cancer screening; about $47 \%$ of providers pointed out that having a separate reimbursement would be necessary to increase oral cancer screening. While this is similar to the $40 \%$ of general dentist in Scotland who felt the National Health Service remuneration system was a barrier to perform oral cancer screening (20), we could not locate data to compare this result to U.S. physicians or dentists. Contrary to previous studies $(8,20)$, only $15 \%$ of respondents in this survey selected time constraints as a barrier to oral cancer screening. This might be due to the difference between FQHCs and private clinic settings.

Results from this survey indicate there is a significant association between PCPs' knowledge level and practice of oral cancer screening as well as willingness to participate in oral cancer screening/prevention programs. This finding underscores the importance of providing up-todate education regarding oral cancer to PCPs. Traditional methods of educational dissemination such as conferences, lectures, journal articles, and mailings were preferred by the respondents over new communication methods such as email, websites, or CD-ROMS. Yet recent systematic reviews found the traditional educational methods did not effectively increase providers' knowledge and change preventive practice behaviors (21). Instead, the evidence indicates that small group discussion, interactive workshops, academic detailing, and reminders are promising in changing PCPs' behaviors (21). There should be effort to plan and adopt effective educational programs considering both the effectiveness and the PCPs' preferences of educational methods. An effective educational approach may need to integrate small group discussions and interactive workshops into the traditional methods of PCP education programs.

Between 1997 and 1999 in Michigan $39.5 \%$ of oral and pharyngeal cancers were detected at early stages (Personal communications with the Michigan Department of Community Health). The findings of this survey indicate that PCPs have a positive attitude about performing oral cancer screening in FQHCs, and would like to take an active part in a network to screen for oral cancer. Their positive attitude and interest in performing oral cancer screenings will be helpful for Michigan to achieve the Healthy People 2010 goal of detecting $50 \%$ of oral and pharyngeal cancers at an 
TABLE 5

Characteristics of primary care providers by knowledge score groups (Bivariate analysis)

\begin{tabular}{|c|c|c|c|}
\hline \multirow{2}{*}{\multicolumn{4}{|c|}{$\begin{array}{c}\text { Characteristics } \\
\text { Demographics and background }\end{array}$}} \\
\hline & & & \\
\hline Physicians (\%) & 60.0 & 40.5 & $<0.01$ \\
\hline Race/Ethnicity (\% white) & 67.7 & 84.2 & 0.087 \\
\hline Age (\% below 40 years) & 43.9 & 38.5 & 0.533 \\
\hline Gender (\% male) & 50.8 & 32.1 & 0.022 \\
\hline \multicolumn{4}{|l|}{ Practice behaviors } \\
\hline Screen for oral cancer ( $\%$ who answered 'yes') & 87.0 & 61.8 & $<0.001$ \\
\hline Referred patient for oral cancer ( $\%$ who answered 'yes') & 72.4 & 60.0 & 0.163 \\
\hline \multicolumn{4}{|l|}{ Attitude/Opinions } \\
\hline Feel adequately trained to detect oral cancer lesions ( $\%$ who answered 'yes') & 47.1 & 27.9 & 0.013 \\
\hline $\begin{array}{l}\text { Oral cancer examinations for adults aged } 40 \text { years and older should be } \\
\text { provided annually (\% who agreed or strongly agreed) }\end{array}$ & 97.2 & 87.2 & 0.052 \\
\hline $\begin{array}{l}\text { Oral cancer examinations for tobacco users should be provided annually } \\
\text { regardless of age (\% who agreed or strongly agreed) }\end{array}$ & 98.6 & 93.1 & 0.357 \\
\hline $\begin{array}{l}\text { Oral cancer examination should be a separate reimbursable } \\
\text { procedure ( } \% \text { who agreed or strongly agreed) }\end{array}$ & 54.3 & 42.4 & 0.494 \\
\hline Would you be willing to participate in a network to promote early & & & \\
\hline screening for oral cancer? (\% who answered 'yes') & 52.9 & 39.3 & 0.024 \\
\hline Total & 44.0 & 56.0 & \\
\hline
\end{tabular}

* P-values are from the Chi-square test.

early stage. Future plans to increase early detection of oral cancer should focus on removing barriers and increase incentives for primary care providers to perform oral cancer screening; which includes providing up-todate education on oral cancer, setting up a referral system, and providing appropriate reimbursement. In developing educational programs, $\mathrm{PCPs}^{\prime}$ preferences for information source should be considered.

\section{Source of Funding}

This project is funded by the $\mathrm{Na}$ tional Institute of Dental and Craniofacial Research / National Institutes of Health Grant DE 14410-03.

\section{References}

1. U.S. Department of Health and $\mathrm{Hu}-$ man Services. Healthy People 2010: Understanding and Improving Health. 2nd ed. Washington, DC: U.S. Government Printing Office, November 2000.

2. Holmes JD, Dierks EJ, Homer LD, Potter BE. Is detection of oral and oropharyngeal squamous cancer by dental health care provider associated with a lower stage at diagnosis? J Oral Maxillofac Surg 2003;61:285-291.

3. Fellona MO, DeVore LR. Oral health services in primary care nursing centers: opportunities for dental hygiene and nursing collaboration. J Dent Hygiene 1999;73:69-77.
4. Bloom B, Gift HC, Jack SS. Dental services and oral health: United States, 1989. Vital Health Stat 10. 1992; No. 183.

5. National Center for Health Statistics. Physician contacts by sociodemographic and health characteristics, 1982-83. Vital Health Stat 10. 1988;No. 161.

6. Fedele DJ, Jones JA, Niessen LC. Oral cancer screening in the elderly. J Am Geriatr Soc 1991;39:920-925.

7. Smart CR. Screening for cancer of the aerodigestive tract. Cancer 1993;72: 1061-1065.

8. Goodman HS, Yellowitz JA, Horowitz AM. Oral cancer prevention: The role of family practitioners. Arch Fam Med 1995;4(7):628-636

9. Canto MT, Horowitz AM, Drury TF, Goodman HS. Maryland family physicians' knowledge, opinions and practice about oral cancer. Oral Oncology 2002;38:416-424.

10. Smith RA, Mettlin CJ, Davis KJ, Eyre $\mathrm{H}$. American Cancer Society guidelines for the early detection of cancer. CA-A Cancer Journal for Clinicians 2000; 50(1):35-49.

11. DiGuiseppi C, Atkins D, Woolf SH. US Preventive Services Task Force. Guide to clinical preventive services. 2nd ed. Baltimore: Williams \&n Wilkins, 1996.

12. Yellowitz J, Horowitz AM, Goodman HS, Canto MT, Farooq NS. Knowledge, opinions and practices of general dentists regarding oral cancer: a pilot survey. J Am Dent Assoc 1998;129:57983.

13. Ahluwalia KP, Yellowitz JA, Goodman HS, Horowitz AM. An assessment of oral cancer prevention curricula in U.S. medical schools. J Cancer Educ 1998; 13:90-5.

14. Baden E. Prevention of cancer of the oral cavity and pharynx. Cancer J Clinicians 1987;37:49-62.

15. Yellowitz JA, Goodman HS. Assessing physicians' and dentists' oral cancer knowledge, opinions and practices. J Am Dent Assoc 1995;126:53-60.

16. Eckert D, Bloom HJ, Ross LS. A review of oral cancer screening and detection in the metropolitan Detroit cancer control program. Prog Clin Biol Res 1982; 83:195-206.

17. Lauritsen JM \& Bruus M. EpiData (version 3). A comprehensive tool for validated entry and documentation of data. The EpiData Association, Odense Denmark, 2003.

18. Lewis CW, Grossman DC, Domoto PK, Deyo RA. The role of pediatricians in the oral health of children: A national survey. Pediatr 2000;206:E84 (http:/ /www.pediatrics.org/cgi/content/ full / 106/6/e84).

19. Scully C, Malamos D, Levers BGH, Porter SR, Prime SS. Sources and patterns of referrals of oral cancer. Br Med J 1986; 293: 599-601.

20. Macpherson LMD, McCann MF, Gibson $\mathrm{J}$, Binnie VI, Stephen KW. The role of primary healthcare professionals in oral cancer prevention and detection. $\mathrm{Br}$ Dent J 2003; 195: 277-281.

21. Sohn W, Ismail AI, Tellez M. Efficacy of educational interventions targeting primary care providers' practice behaviors: An overview of published systematic reviews. J Public Health Dent 2004;64:164-72. 